\title{
Thermal Error Prediction and Compensation of YK3610 Hobbing Machine Based on BP Neural Networks
}

\author{
Qianjian Guo ${ }^{*}, 1$, Rufeng $\mathrm{Xu}^{1}$ and Xiaoni $\mathrm{Qi}^{2}$ \\ ${ }^{I}$ School of Mechanical Engineering, Shandong University of Technology, Zibo 255049, China \\ ${ }^{2}$ School of Transportation and Vehicle Engineering, Shandong University of Technology, Zibo 255049, China
}

\begin{abstract}
In this study, error compensation technology was proposed to reduce thermal errors of a gear hobbing machine, and one experiment was carried out to verify the compensation effect. Different thermal sources were used as modeling variables, and a prediction model of thermal errors was presented based on back propagation (BP) neural networks. In order to solve local minimum problem of BP neural networks, ant colony algorithm was used for training its link weights. Finally, one test system was developed based on the presented model, and an experiment was fulfilled. The result shows that prediction performance of the model is very well, and the residual error is less than $5 \mu \mathrm{m}$ after compensation.
\end{abstract}

Keywords: Thermal error prediction, Gear hobbing machine, BP neural networks, thermal error compensation.

\section{INTRODUCTION}

Hobbing is the most widely used ways to process cylindrical gears, and gear hobbing machine account for 45 percent of total gear cutting machines [1]. In order to improve the machining accuracy of gear hobbing machine, there are mainly two methods. The method commonly used is improving the accuracy of machine itself, such as the following methods: (1) Zero-drive chain [2], which removes all links of mechanical transmission, the gear hob and worktable are driven directly by motors. Because of the removing of mechanical parts, such as gears, bearing and so on, the errors induced by transmission system, such as the backlash and the meshing error, are deleted, which greatly improves machine accuracy; (2) High-precision machine structure [3], such as high-precision linear guides, linear bearings, ball screws and so on, which improves machine accuracy by using high-precision machine structure; (3) Intelligent controlling of hobbing process [4], which inspects processing quality and machine conditions online and adjusts processing parameters in real-time. The second method is error compensation, such as thermal error compensation [5], tool wear compensation [6], geometric error compensation [7] and so on. Among these methods, thermal error prediction and compensation is most effective.

In order to improve the machining accuracy of gear hobbing machine, a lot of studies focused on thermal error prediction and compensation has been presented recently, such as artificial neural networks $[8,9]$, GA-BPN method [10], project pursuit regression method [11], information fusion method [12], and so on. In this study, BP neural network algorithm was applied to predict thermal errors of a YK3610 hobbing machine, and ant colony algorithm was

*Address correspondence to this author at the School of Mechanical Engineering, Shandong University of Technology, Zibo 255049, China;

Tel: +86-2781723; E-mail: guoqianjian@163.com used to train the link weights of BP neural network model, which overcomes the local minimum problem of BP neural networks and improves the prediction performance of thermal error modeling. Finally, one test system was developed based on the presented model, and an experiment was fulfilled. During experiment, thermal errors of the gear hobbing machine were compensated online, and the machining accuracy is improved.

\section{EXPERIMENTS AND DATASETS}

\subsection{Experimental Details}

One measurement experiment was carried out on a $\mathrm{NC}$ gear hobbing machine, which was shown in Fig. (1). In order to measure the temperature of different thermal sources, 10 temperature sensors were mounted on this gear hobbing machine, and the positions of these sensors were as follows: one sensor was used to measure bed temperature, two sensors were used to measure guideway temperature, another two sensors were used to measure hob spindle temperature, one sensor was used to measure column temperature, and two sensors were used to measure workpiece spindle temperature, one sensor was used to measure feed plate temperature, and one sensor was used to measure coolant temperature.

In order to measure thermal deformations of the spindle in $\mathrm{X}$ direction (Thermal errors of the spindle in radial direction), a displacement sensor was installed on the tool turret, which was shown in Fig. (2). As was shown, error signals were collected by displacement sensor and sent to signal processing unit (SPU). After amplifying and AD conversion, error signals were sent to PC finally.

In order to simulate the working cycle of YK3610 hobbing machine, an experiment was carried out, the hob spindle speed was set as $1000 \mathrm{r} / \mathrm{min}$, and the workpiece spindle speed was set as $100 \mathrm{r} / \mathrm{min}$, the coolant was on, but no gear was machined really. First, the machine warmed up 
for 4 hours, and then the machine ran for another 2 hours. Finally, the machine was cooled down for 6 hours. As was shown in Fig. (3), temperature of different thermal sources changed continuously with the working cycle. At beginning, the machine was warmed up, and temperature of different thermal sources increased gradually, then the machine reached thermal equilibrium, and the temperature no longer increased. Finally, when the machine was cooled down, the temperature decreased as expected. As was shown in Fig. (4), thermal errors of the machine were well consistent with the working cycle too.

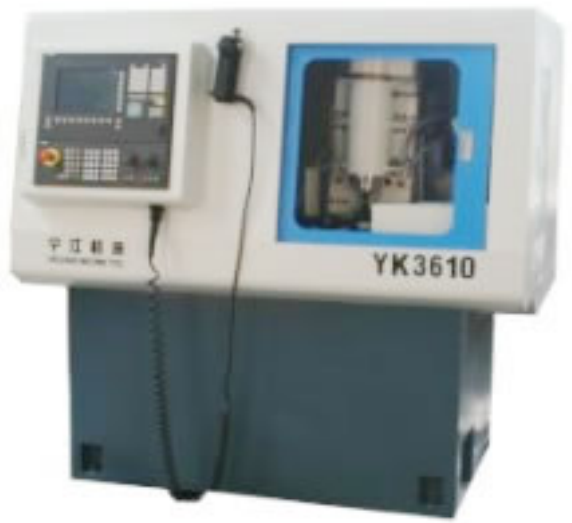

Fig. (1). Gear hobbing machine used in experiment.

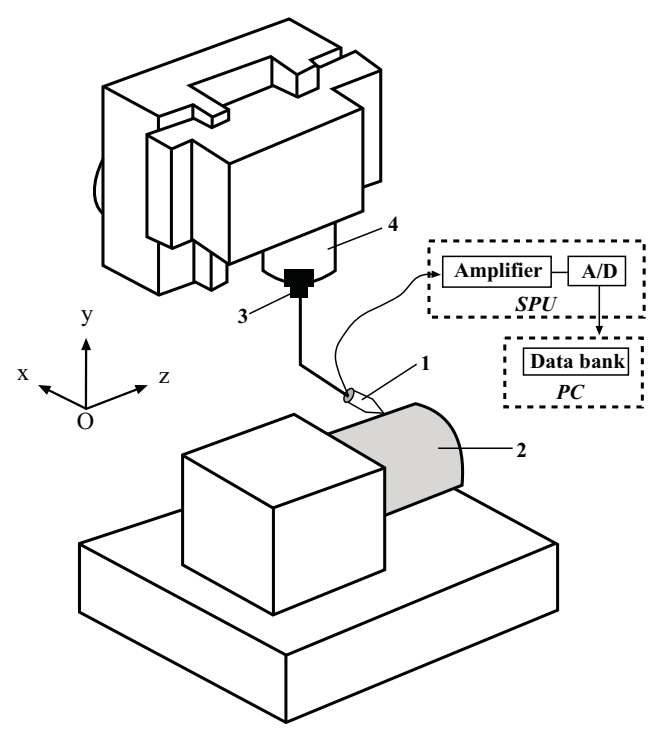

Fig. (2). Thermal errors measurement experiment.

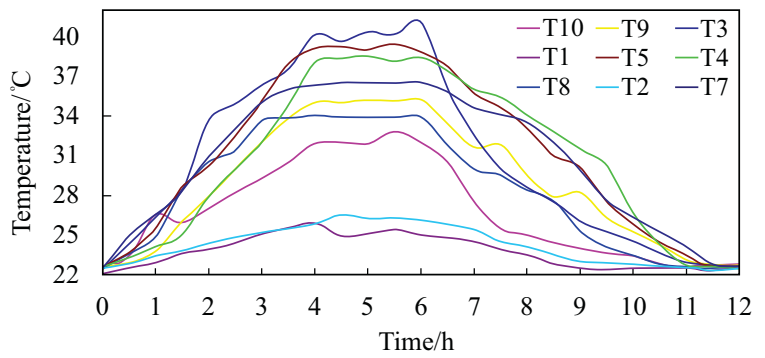

Fig. (3). Temperature of different thermal sources.

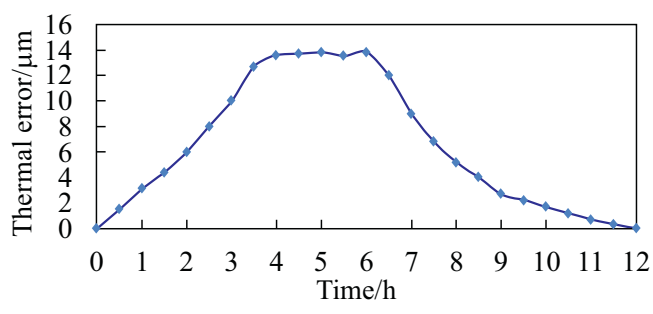

Fig. (4). Thermal errors of YK3610 hobbing machine.

\subsection{Thermal Error Prediction}

Artificial intelligent neural networks are composed of many neurons, which is a dynamic system with nonlinear mapping ability. In recent years, neural networks have been introduced into many research fields successfully and solved many nonlinear problems. Among different neural network methods, BP neural networks are most widely used, because it has a simple structure. Besides input layer and output layer, BP neural network has hidden layer. Nodes in same layer have no coupling, and sigmoid is the commonly used transfer function of different neurons. Even inputs of BP neural networks change linearly, outputs of which maybe nonlinear linear. According to these characteristics, BP neural networks are widely used in prediction fields of complicated systems.

The training process of BP neural network is composed of 2 steps. The first one is forward propagation, during which, inputs are dealt with input layer, hidden layer and output layer. The second one is back propagation. If outputs of BP neural networks are not satisfied, error value will propagate backward, and the link weights of different layers will be adjusted until the error value decreased to its threshold. During the application of BP neural network, local minimum is a commonly occurred problem, which induces to bad stability and low convergence rate.

In order to improve network performance, increasing of hidden nodes, decreasing of momentum and learning rate are commonly used ways. However, these methods induce to high computation cost, and new training method is still needed. In this paper, ant colony algorithm was proposed to train the link weights of BP neural networks.

In a colony of ants, individual behavior is adaptive and robust, and the ants are not controlled by any regulations. In the colony, simple individual behaviors converge into complex social behaviors by local interacting and communicating, these characteristics promote the application of ant colony algorithm in different fields. Among these applications, ant colony optimization is most successful, which was proposed by Marco Dorigo and applied to solve scheduling problem, traveling salesman problem and so on.

The structure of ant colony is as follows: some agent ants, using of memory and decision, collecting and distributing learning strategies. Ant colony algorithm is proposed to simulate the behavior of searching food, and ant colony can find the shortest path to the food sources. Pheromone laid on the ground is the mediator of selecting behavior. When ant colony moving, pheromone intensity will affect its decisions.

In this study, the structure of BP neural networks was presented firstly, and ant colony was applied to simulate the propagating of input signals among different layers. The ant 
colony was propagated by a stochastic transfer function locally, and only local information was used for stochastic decision. Therefore, local minimum problem was solved.

According to above analysis, a BP neural network was presented, which is applied to predict thermal errors of YK3610 hobbing machine. 10 neurons were applied to simulate 10 thermal sources, which denote different temperature variables. 1 neuron was applied to simulate thermal errors. No perfect rules can be used to determine hidden node number. Generally, a large number is chosen firstly. If error demand is not satisfied, the number will be adjusted until prediction error reaches its threshold. In this study, Kolmogorov method was proposed to determine nodes number of the hidden layer, and which was set as 21 finally. The structure of presented BP neural network was shown in Fig. (5). Then, fifty training samples were collected by different sensors mounted on the gear hobbing machine. Parameters of BP neural networks were shown in Table 1. Finally, ant colony algorithm was applied to adjust the 231 link weights, and the flowchart of adjusting process was shown in Fig. (6).

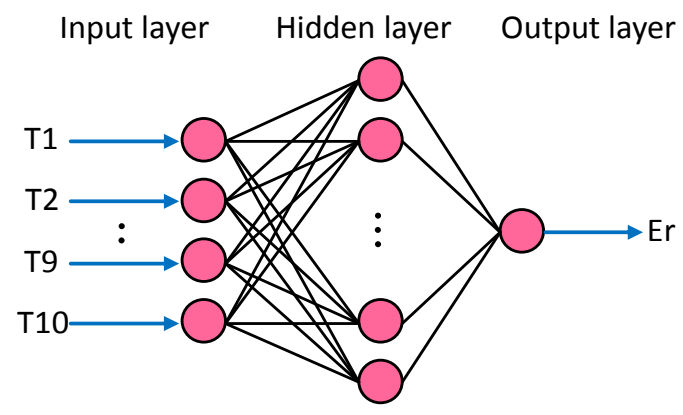

Fig. (5). BP neural network structure.

Table 1. Parameters of BP neural networks.

\begin{tabular}{|c|c|}
\hline Parameters of BP Neural Network & Value \\
\hline \hline Structure of BP neural network & $10-21-1$ \\
\hline Original weight & Random $(0,1)$ \\
\hline Training rate & 0.02 \\
\hline Momentum rate & 0.5 \\
\hline Adjusting coefficients & 0.8 \\
\hline
\end{tabular}

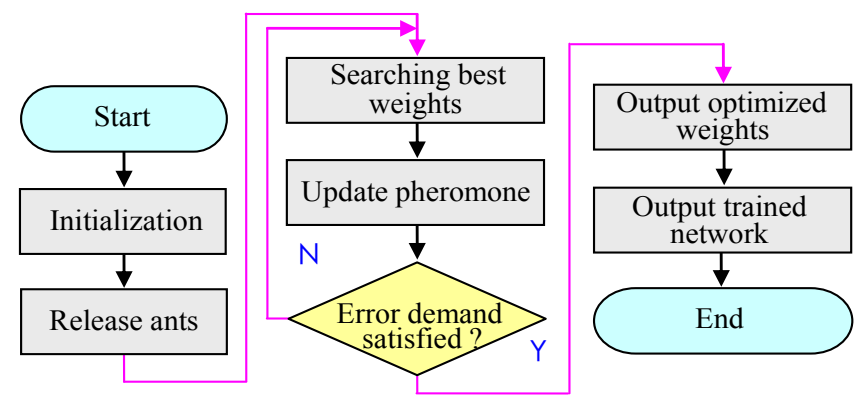

Fig. (6). Flowchart of weights adjusting process.

In this study, link weight between input and hidden layer was represented by $w_{i j}(i=1,2, \ldots 10 j=1,2, \ldots, 21)$, bias of hidden node was represented by $\emptyset_{j}(j=1,2, \ldots, 21)$, link weight between hidden and output layer was represented by $u_{j}(j=1,2, \ldots, 21)$, bias of output node was represented by $\theta$. Pheromone of path $i-j$ was represented by $s_{i j}(t)$, pheromone of path $j$-output layer was represented by $s_{j}(t)$, which was applied to simulate pheromone intensity of ant colony. Training process of BP neural network was as follows:

(1) At beginning, ten ants were allocated on different input nodes, pheromone original value of path $i-j$ was represented by $s_{i j}(0)$, pheromone original value of path j-output layer was represented by $s_{j}(0)$.

(2) All $w_{i j}$ formed one class $L_{w}$, all $u_{j}$ formed one class $R_{u}$. Ant colony selected element from $L_{w}$ and $R_{u}$ based on intensity ratio of the pheromone. Therefore, selecting probability of an element in $L_{w}$ and $R_{u}$ was computed as follows:

$$
p_{w_{i j}}(t)=\frac{s_{L_{w}}(t)}{\sum s_{L_{w}}(t)} \quad p_{u_{j}}(t)=\frac{s_{R_{u_{j}}}(t)}{\sum s_{R_{u}}(t)}
$$

where, $S_{L w}(t)$ represented element pheromone value in $L_{w}, S_{R u}(t)$ represented element pheromone value in $R_{u}$. In the end, element with largest probability was selected as optimized weight.

Then, different link weights were adjusted as following:

$$
\begin{aligned}
& s_{i j}(t+\Delta t)=\alpha \cdot s_{i j}(t)+\sum_{n=1}^{10} \Delta s_{i j}^{n} \\
& s_{j}(t+\Delta t)=\alpha \cdot s_{j}(t)+\sum_{n=1}^{10} \Delta s_{j}^{n}
\end{aligned}
$$

where, $\alpha$ represented pheromone attenuation coefficient, $\Delta \mathrm{t}$ represented time step. $\Delta s_{i j}^{n}$ represented pheromone of path $i-j, \Delta s_{j}^{n}$ represented pheromone of path j-output layer, which were computed as follows:

$$
\begin{aligned}
& \Delta s_{i j}^{n}=Q / e_{w_{i j}} \\
& \Delta s_{j}^{n}=Q / e_{u_{j}}
\end{aligned}
$$

where, Q represented a constant, which was applied to adjust pheromone growth rate. $e_{w_{i j}}$ represented maximum error of different hidden nodes, $e_{u_{j}}$ represented maximum output error. $e=\max _{l=1}^{m}\left|Y_{l}-O_{l}\right|$, and $Y_{l}$ represented expected value of different neural cells, $O_{l}$ represented practical value of different neural cells. When pheromone intensity reached its threshold, error demand was satisfied, optimized weights were output.

\section{COMPENSATION EXPERIMENT}

In order to verify the prediction performance of proposed model, an experiment system was developed in this paper, which was shown in Fig. (7). During experiment, temperature of different thermal sources was measured by temperature sensor, and thermal error was measured by displacement sensor. Then, signals of different sensors were processed by amplifier, $\mathrm{AD}$ board, and digital signal processor and so on. Finally, 
processed signals were sent to PC by serial port. According to these processed signals, thermal error model was presented, and which was sent to digital signal processor. Based on the presented model, compensation value was computed, and the results were sent to the controller of YK3610 hobbing machine. After compensation value was added to the servo loop of YK3610 hobbing machine, compensation was fulfilled in the end.

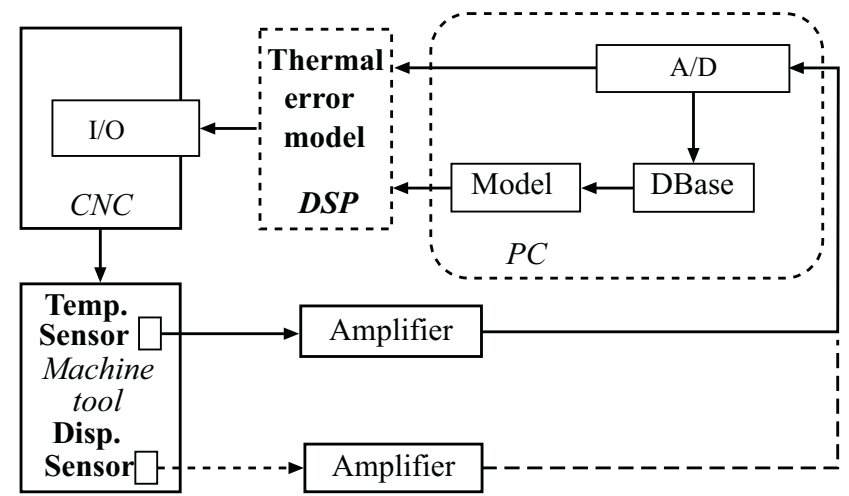

Fig. (7). Compensation experiment system.

An experiment was carried out with the developed system, which was used to test the prediction performance of presented model. Ten cylindrical gears were processed with YK3610 hobbing machine. 45 steel was selected as part material. Hob spindle speed was set as $1000 \mathrm{r} / \mathrm{min}$, and workpiece spindle speed was set as $100 \mathrm{r} / \mathrm{min}$. In the experiment, thermal errors were measured by displacement sensor, and prediction results were presented by proposed model. Measurement and prediction results were shown in Fig. (8). As was shown, prediction performance of proposed model was very well, model residual error was less than $4.5 \mu \mathrm{m}$, experiment system developed in this study is very effective.

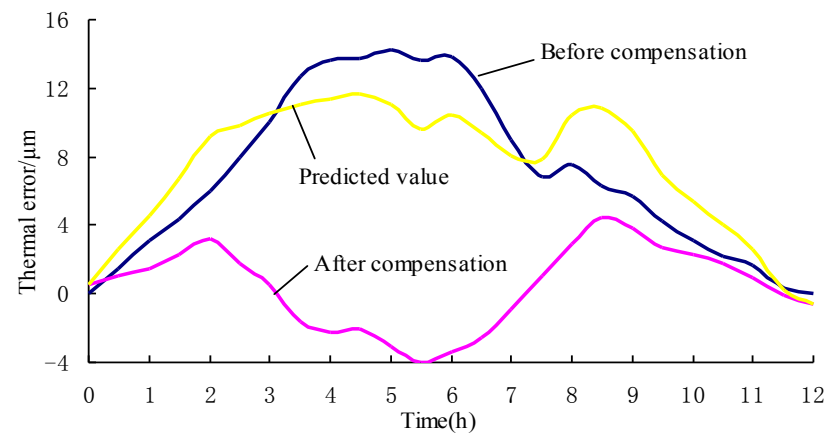

Fig. (8). Compensation and prediction results.

\section{CONCLUSION}

In this study, a new BP neural network model was presented, which was used to predict thermal errors of YK3610 hobbing machine. Prediction performance of proposed model was very well. During experiment, thermal errors reduced from $16 \mu \mathrm{m}$ to $4 \mu \mathrm{m}$, machining accuracy of YK3610 hobbing machine was improved significantly.

\section{CONFLICT OF INTEREST}

The authors confirm that this article content has no conflict of interest.

\section{ACKNOWLEDGEMENTS}

We thank Jianguo Yang for critical reading of the manuscript. This project was supported by National Natural Science Foundation of China (No. 51305244), Natural Science Foundation of Shandong Province (No. ZR2013EEL015), Project of Shandong Province Higher Educational Science and Technology Program (No. J11LD24).

\section{REFERENCES}

[1] R.A. Liu, and G.B. Zhang, "Deveolopment trend of gear hobbing machine and gear hobbing technology", Modern Manuf. Technol., vol. 24, pp. 84-86, November 2003.

[2] A. Muller, and T. Hufnagel, "Model-based control of redundantly actuated parallel manipulators in redundant coordinates", Robot. Auton. Syst., vol. 60, pp. 563-571, April 2012.

[3] S.L. Wang, Y. Yang, X.G. Li, J. Zhou, and L. Kang, "Research on thermal deformation of large-scale computer numerical control gear hobbing machines", J. Mech. Sci. Technol., vol. 27, pp. 1393-1405, May 2013.

[4] B. Karpuschewski, H.J. Knoche, and M. Hipke, "Gear fishing by abrasive processes", CIRP Ann.-Manuf. Technol., vol. 57, pp. 621-640, February 2008.

[5] S. Yin, Y. Ren, J. Zhu, S. Yang, S. Ye, and Y. Guo, "Real-time thermal error compensation method for robotic visual inspection system", Int. J. Adv. Manuf. Technol., vol. 75, pp. 933-946, October 2014.

[6] J. Z. Li, L. Xiao, H. Wang, H.L. Yu, and Z.Y. Yu, "Tool wear compensation in 3D micro EDM based on the scanned area", Prec. Eng., vol. 37, pp. 753-757, July 2013.

[7] G.S. Chen, X.S. Mei, and H.L. Li, "Geometric error modeling and compensation for large-scale grinding machine tools with multi-axes", Int. J. Adv. Manuf. Technol., vol. 69, pp. 2583-2592, December 2013.

[8] Y.J. Hu, Y.Q. Wang, G.L. Zhao, Y. Wang, and X.H. Yuan, "Featurebased modeling of automobile gears and manufacturing resources for virtual manufacturing", Int. J. Adv. Manuf. Technol., vol. 55, pp. 405419, July 2011.

[9] W. Xiong, X.H. Xian, and L.J. Zhang, "Application of neural network integration in fault diagnosis", Open Mech. Eng. J., vol. 8, pp. 81-84, March 2014.

[10] H. Wang, H.T. Zhang, Q.J. Guo, X.S. Wang, and J.G. Yang,' Thermal error optimization modeling and real-time compensation on a CNC turning center", J. Mater. Process. Technol., vol. 207, pp. 172-179, January 2008.

[11] Q.J. Guo, and J.G. Yang, "Application of project pursuit regression to thermal error modeling of a CNC machine tool", Int. J. Adv. Manuf. Technol., vol. 55, pp. 623-629, July 2011.

[12] Q. Guo, R. Xu, C. Mao, H. Xu, and J. Yang, "Application of information fusion to volumetric error modeling of CNC machine tools", Int. J. Adv. Manuf. Technol., In press, December 2014. 